\title{
Studien über die giftigen Leibessubstanzen von Staphylococcus aureus.
}

\author{
I. Mitteilung. \\ Von \\ Shunjiro Inoue. \\ (井上俊次 郎) \\ (Aus dem Bakteriologischen Institut der Tohoku Kaiserlichen \\ Universität zu Sendai. \\ Direktor: Prof. Dr. M. Kuroya.)
}

Seitdem Burn et ${ }^{1{ }^{2)}}{ }^{\text {3) }}$ durch die Bebrütung von Staphylokokken in $\mathrm{CO}_{2}-$ Atmosphäre stark giftige Toxine gewann, haben sich die Studien in dieser Richtung ausserordentlich erweitert, besonders die nacheinander auftretenden therapeutischen Berichte im Hinblick auf die Toxine und Antitoxine sind in der Tat erstaunenswert. Da klar ist, dass bei der Staphylokokkeninfektion der Heilungsvorgang nicht nur auf die Antitoxinbildung zurückgeführt werden kann, sondern mit den bakteriziden Antikörpern eine enge Abhängigkeit besteht, so bieten uns die Untersuchungen über spezifische Substanzen dieser Bakterien eine grosse Aufgabe dar.

Seit der Bekanntmachung von Avery und Heidelberger ${ }^{4)}$, dass von Pneumokokken von verschiedenen Typen die typenspezifischen Kohlehydrate abtrenmbar waren, sind biochemische wie immunologische Untersuchungen über die bakterienkörperbildenden spezifischen Substanzen eifrig vorgenommen worden.

Die Isolierung der wirksamen Fraktion aus Staphylokokken wurde im Jahre 1934 von Julianelle und Wieghard ${ }^{5) 6}$ beabsichtigt. 18-24 stündige Staphylokokkenkulturen von A und B Typen, von denen der erste die virulenten Stämme, der letztere dagegen die avirulenten enthielten, wurden in $\mathrm{N} / 16 \mathrm{HCl}$ aufgeschwemmt, nach 20 Minuten langem Erhitzen im Wasserbad von $100^{\circ}$ gekühlt und zentrifugiert, überstehende Flüssigkeit mit 40 proz. $\mathrm{NaOH}$ zweimal behandelt und durch Zentrifugieren fällbares Bakterienprotein-wie er es bezeichnete-abgetrennt. $\mathrm{Zu}$ der hiernach überstehenden Flüssigkeit, nachdem sie 
bei starker Alkalisierung keine Fällung mehr brachte, kamen Natriumazetat und 2-4 Volumen Alkohol. Nach dem Stehenlassen im Eisschrank wurde diese zentrifugiert, der Niederschlag mit 50 proz. Trichloressigsäure und Alkohol dreimal wiederholt behandelt, dialysiert und eingeengt, durch Zusatz von 10 Volumen Azeton zur Fällung gebracht, mit abs. Alkohol und Äther gewasehen und so weissliches Pulver von Kohlenhydraten gewonnen. Diese Autoren haben also gefunden, dass Typus A und B typenspezifsche Kohlenhydrate enthielten, die chemisch voneinander eine abweichende Zusammensetzung besassen, und ferner, dass auch das aus Bakterien gewonnene Nukleoprotein eine Gruppenspezifitüt besass.

Yat ${ }^{7)}$ wandte zur Reindarstellung des Toxins, das bei der Eiweissreaktion negativ ausfiel, aus dem Filtrate der Bouillonkulturen von Staphylococcus aureus $\mathrm{Al}(\mathrm{OH})_{3}-$ Methode, aber auch zu der des Bakterieneiweisses das Verfahren von Wooldridge an und bestätigte die Möglichkeit der Isolierung derselben. Die aktive Immunisierung mit diesem Toxin führte bei Kaninchen, wenn auch leichtgradig, zur Bildung von Antitoxin.

Robert ${ }^{8)}$ sagte in seinem Versuche: Das hämolytische Prinzip und die Fähigkeit des Toxins, epi- und intrakutane Reaktion zu erzeugen, waren nicht in der durch Ammonsulfat ausgesättigten Eiweissfraktion des Toxins, sondern in der alkoholfällbaren, wasserlöslichen Fraktion enthalten; vermutlich gehören sie der Kohlenhydratfraktion an.

Kodama und Nis iy a $\mathrm{ma}^{9}$ befassten sich mit der Hitzeempfindlichkeit der spezifisch toxischen Gruppe und der Flockungseigenschaft des Staphylotoxins und mit der Trennung dieser Anteile. Beim Erhitzen auf $65^{\circ}$ während $30 \mathrm{Mi}-$ nuten blieb nur $1 / 2$ bis $1 / 3$ der Flockungsfähigkeit erhalten. Vom Toxin überstand $1 / 8$ eine Erhitzung auf $95^{\circ}$ und $1 / 20$ auf $100^{\circ}$. Dieses hitzeinaktivierte Toxin war jedoch noch antigenetisch für Tiere. Als beste Fällungsmittel für reines Toxin wurden Methylalkohol und Metaphosphorsäure angeführt. In der Meinung, dass die Methode von Julianelle und Wieghard zu stark war, versuchte $\operatorname{Verwe} \mathrm{y}^{\mathrm{I0})}$, auf mehr gemässigte Weise spezifische Substanzen zu extrahieren. 24stündige Rinderherzbouillonkulturen vom Typus A-Stamm wurden zentrifugiert, die angesammelte Bakterienmasse verrieben, in destilliertem Wasser aufgeschwemmt und mittels $\mathrm{N} / .10 \mathrm{HCl}$ und 50 proz. Trichloressigsäure Fraktionen A, B, C und $\mathrm{R}$ abgesondert gewonnen. Auf Grund der chemischen und der serologischen Befunde dieser Fraktionen ging hervor, dass die Typus A-Stämme typenspezifische und antigenes Protein in sich einschliessen, dass in den als Nebenprodukte extrahierten Anteilen, wie früher Julianelle und Wieghard mitteilten, typenspezifisches Kohlenhydrat vorhanden war, von dem sich das von Typus B-Stamm isolierte serologisch wie immunologisch unterschied.

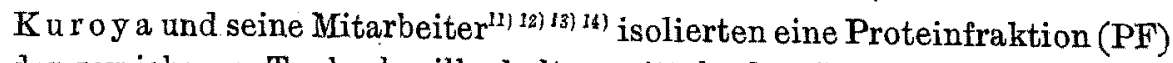
aus der zerriebenen Typhusbazillenkultur mittels der Säurefällung und eine Kohlenhydratfraktion (CF) aus deren überstehenden Flüssigkeit mittels der Alkoholfällung. Sie untersuchten weiter verschiedene chemische und biologische Eigenschaften der beiden Fraktion, d.h. die Protein- und Kohlehydratreak- 
tionen, N- und P-Gehalt, die Menge der reduzierenden Substanzen nach der Hydrolyse, die Toxität und die in einer schwach und einer hoch virulenten Typhusbazillenkultur enthaltene Menge der beiden Fraktionen.

Neuerdings extrahierten sie aus einer stark virulenten Typhusbazillenkultur eine toxische Kohlenhydratfraktion mittels einer Erhitzungsmethode in schwach saurer Reaktion und isolierten aus der letzteren einen mukoidähnlichen Polysaccharid-Eiweisskomplex, welcher nicht fällbar in schwach sauerer Reaktion, stark tocisch für Mäuse und zugleich in vitro und vivo stark antigenetisch war.

Yamamoto ${ }^{15) 16)}$ bestätigte alle diese Befunde von Kuroya und seinen Mitarbeitern in der Untersuchung mittels eines schwach und eines hoch virulenten Stammes von Mäusetyphusbazillen.

Andererseits studierte $\mathrm{Suganum} \mathrm{a}^{\text {17) 18) 19) 20) }}$ die toxische Leibesubstanzen von hämolytischen Streptokokken und isolierte aus ihrer Kultur eine stark toxische, säurelösliche Eiweissfraktion.

Der Verfasser hiermit unternahm, die toxische Leibessubstanzen von Staphylokokken mittels oben erwähnter verschiedener Methoden zu isolieren und deren chemische und biologische Eigenschaften zu untersuchen.

\section{Darstellung des nativen Toxinextraktes.}

\section{Verwendete Stämme und ihre Züchtung.}

Nachdem 16 Staphylokokkenstämme, die aus Fällen von Otitis media purlenta acuta, Mastoiditis acuta, Ohrkarbunkel, Osteomyelitis acuta und Halsabscess isoliert wurden, durch die Untersuchungen über 1) Form, Färbbarkeit durch Gramsches Verfahren 2) Kolonien- sowie Hämolysinbildung auf der Blutagarplatte 3) Koagulationsfähigkeit an Zitratplasma 4) Spaltungsreaktion in Glukosaminlösung und 5) Virulenz für Mäuse, als Staphylococcus aureus, festgestellt wurden, wählte Verfasser darunter solch einen Stamm zum Versuche aus, der sich in den obengeschilderten Reaktionen am besten bewährte.

Dieser Stamm war bei Ohrkarbunkel isoliert, glattförmig, seine Toxität betrug für Mäuse 1/10 mg. Auf gewöhnlichen Nährböden, die aus Pferdebouillon und Polypepton bestanden, wurden die Bakterien bei $37^{\circ} \mathrm{C} 18$ Stunden lang gezüchtet.

2. Extraktion des nativen Toxinextraktes.

Um die toxischen Leibessubstanzen zu isolieren, wurden folgende verschiedene Methoden ausgeführt:

i) durch 15 Minuten langes Erhitzen bei pH 5,0 und 7,0.

ii) durch 30 Minuten langes Erhitzen bei pH 5,0 und 7,0.

iii) durch 1 Stunde langes Erhitzen bei pH 5,0 und 7,0. 
An dieser Stelle soll aber fürs erste die (i)-Methode besprochen werden.

$21 \mathrm{~g}$ (feuchtes Gewicht) dieser Bakterien wurde zuerst in destilliertem Wasser ( $20 \mathrm{ccm}$ für $1 \mathrm{~g}$ Bakterien) homogen aufgeschwemmt, mittels 5\% Essigsäure zu pH 5,0 gebracht und unter ständigem Umrühren im Wasserbad von $100^{\circ}$ für 15 Minuten lang erhitzt und zentrifugiert, der Niederschlag nochmals in $420 \mathrm{ccm}$ dest. Wasser aufgeschwemmt und nach Ansäurung ( $\mathrm{pH} 5,0)$ in ganz gleicher Weise wie oben behandelt. Nach noch einmal wiederholter Manipulation, wurden die Flüssigkeiten zu pH 7,0 gebracht, mittels einer "Sharpless" Zentrifuge von 14000 Umdrehungen pro Minute abgeschleudert, und so der sog. native Toxinextrakt gewonnen. Andere fast in gleicher Menge kultivierten Staphylokokken wurden dagegen durch Erhitzen bei $\mathrm{pH}$ 7,0 extrahiert. Das Verfahren bei (ii) und (iii) war ganz dasselbe wie bei (i), abgesehen von der Zeitdauer des Erhitzens. Wasser.

iv) durch Verreiben der Bakterien in neutralem, destilliertem

Nachdem die Bakterienmasse (F.G.ca. $53 \mathrm{~g}$ ) in einem Mörser etwa 6 Stunden lang verrieben wurde, kam diese in destilliertes Wasser (10 ccm für $1 \mathrm{~g}$ Bakterien) zur Suspension, wurde in $\mathrm{pH} 7,0$ einige Zeit umgerührt und dann zentrifugiert. Der Niederschlag wurde nach Umrührung in gleicher Menge Wasser wieder bei $\mathrm{pH} 7,0$ abgeschleudert. Nach noch einmaligem Wiederholen dieses Verfahrens wurden gewonnene Flüssigkeiten angesammelt, neutralisiert, mittels schnell umdrehender Zentrifuge abgeschleudert und zum Schluss wieder zu pH 7,0 gebracht. Die in solcher Weise gewonnene Flüssigkeit bezeichnete man auch nativer Toxinextrakt.

Isolierung der Fraktionen aus dem Extrakt.

Jeder native Extrakt wurde in folgender Weise behandelt, um daraus toxische Fraktionen zu isolieren.

1. Isolierung der säurepräzipitierenden Fraktion.

Der Extrakt wurde bei $\mathrm{pH} 7,0$ bis auf ungefähr $1 / 20$ des Volumens eingeengt, zu pH 5,0 angesäuert, nach Fällung mit 3 Volumen Alkohol und dem darauf folgenden Stehenlassen für eine Nacht im Eisschrank abgeschleudert. Nach der Entfernung des in Neutralreaktion unlöslichen Anteils, der nur beim (iv)-Verfahren entstand, wurde der Niederschlag mit wenig destilliertem Wasser zur Lösung gebracht, mittels Essigsäure bei pH 3,2 gefällt und abzentrifugiert. Nach 5maliger 


\section{Tabelle 1.}

Bakterienaufschwemmung

Ansäuerung mit $\mathrm{CH}_{3} \mathrm{CO}_{2} \mathrm{H}(\mathrm{pH} 5,0)$, 15 minütiges Erhitzen auf $100^{\circ}$, Zentrifugieren

Niederschlag

Zusatz von $420 \mathrm{ccm}$ Aq. dest.,

15 minütiges Erhitzen bei pH 5,0

auf $100^{\circ}$, Zentrifugieren



Aufschw. in $420 \mathrm{ccm}$

Aq. dest., 15 minütiges

Erhitzen auf $100^{\circ}$,

Zentrifugieren

$\underset{\downarrow}{\downarrow} \frac{1}{\downarrow}$ Niederschlag $\quad$ Überstehende Flüssigkeit (3)

(Bakterienrest)

Niederschlag

Auflösung in einer geringeren

Menge Aq. dest. (pH 7,0), wieder

Ansäuerung mit Eisessig ( $\mathrm{pH} \mathrm{3,2)}$

$\downarrow$

Zentrifugieren

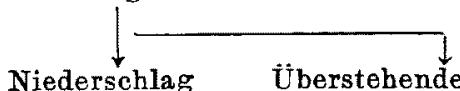

Niederschlag Überstehend

Menge Aq. dest., Ansäuerung

mit Eisessig ( $\mathrm{pH} 3,2)$

$$
\downarrow
$$

Zentrifugieren

Noch zweimaliges ṫberstehende

Wiederholen der -Flüssigkeit $\left(2^{\prime}\right)$

obigen Manipulation

Niederschlag

Waschen mit Alkohol und

Äther, Trocknen und

Pulverisieren

(Rohe "PF")

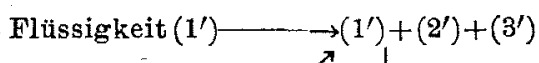

and $\mathrm{CH}_{3} \mathrm{CO}_{2} \mathrm{H}(\mathrm{pH} \mathrm{5,0)}$

Zusatz von 3 Vol. abs. Alkohol,

Belassen im Eisschrank eine

Nacht lang

"Sharples "-Zentrifuge

(14000 Umdrehungen pro Min.)

Konzentration

$\downarrow$

Zentrifugieren

Ưberstehende Flüssigkeit

$(1)+(2)+(3)$

Neutralisation

(Ut) 
Reinigung durch Säurefällung und Aufösung in destilliertem Wasser wurde der letzte Niederschlag mit Alkohol und Äther gewaschen, getrocknet und in Form von Pulver aufbewahrt. Dieses Präparat bezeichnete man einfach rohe „PF.“ Der bei pH 7,0 unlösliche Anteil löste sich in Alkohol gar nicht, jedoch mit Hilfe von Chloroform unter leichtem Erhitzen sehr gut, so dass er darauf einer wachsartigen Substanz angehörig zu sein schien.

\section{Isolierung der süurelöslichen Fraktion.}

Die angesammelte Flüssigkeit wurde bei $\mathrm{pH} 7,0$ und Unterdruck konzentriert. Nachdem man die übrig bleibende, mittels Essigsäure präzipitierende Substanz der PF hinzufügte, versetzte man dann die Flüssigkeit mit 3 Volumen abs. Alkohol und hielt sie eine Nacht über im Eisschrank, zentrifugierte sie ab, wusch sie mit Alkohol und Äther und bewahrte sie schliesslich in Gestalt von weissem Pulver auf. Diesbezügliches Verfahren veranschaulicht folgende Tabelle (Tabelle 1).

\section{Toxitätvon PFund CF.}

Die in solcher Weise hergestellten Fraktionen wurden in $\mathrm{NaCl}-$ Lösung gelöst und den Mäusen intraperitoneal injiziert, um ihre Toxität zu untersuchen.

Wie man aus Tabelle 2 ersieht, verhielt sich die PF, die aus verriebenen Bakterien bei $\mathrm{pH} 7,0$ unerhitzt extrahiert wurde, am giftigsten (M.L.D. $=2 \mathrm{mg}$ ), dem nächst die bei $\mathrm{pH} \mathrm{7,0} \mathrm{für} 15$ Minuten lang erhitzte; bei den anderen nahm die Toxität auffallend ab. Die bei pH

$$
\text { Tabelle } 2 .
$$

Giftigkeit der in verschiedener Weise isolierten Fraktionen.

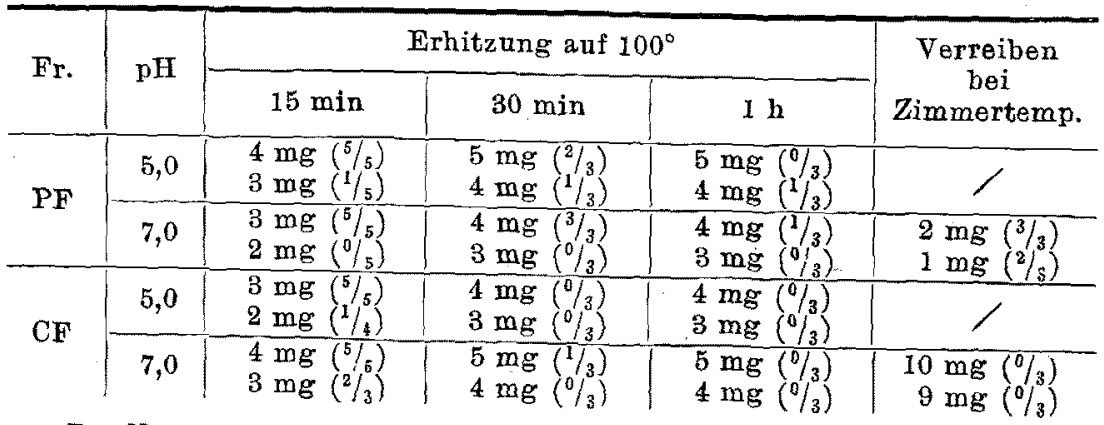

Der Nenner zeigt die Gesamtzahl der gebrauchten Tiere, der Zähler die Zahl der verstorbenen unter ihnen. 
5,0 15 Minuten lang behandelte CF zeigte stärkste Toxität (M.LD.= $3 \mathrm{mg}$ ), dem nächst die bei pH 7,0 15 Minuten lang behandelte, bei den anderen war sie nur sehr schwach; insbesondere war die durch Methode (iv) gewonnenne CF nicht in der Lage, selbst mit einer Dosis von 10 mg Mäuse zu töten. Daraus geht klar hervor, dass zur Gewinnung von giftiger PF die Behandlung der Bakterien ohne Erhitzen und zu der von giftiger CF ein 15 minutiges Erhitzen bei pH 5,0 am geeignetsten sind.

\section{Ausbeuteder PF und CF.}

Die quantitativen Verhältnisse zwischen den gesamten Trockenbakterien und den gewonnenen Fraktionen sind aus Tabelle 3 ersichtlich. Es war zwar nicht möglich, wie die Tabelle dartut, diese Fraktionen quantitativ zu gewinnen, aber es könnte dennoch durch die vergleichenden Untersuchungen der Fraktionen beziiglich ihrer Ausbeute ungefähr folgende Tatsache herausgezogen werden.

$$
\text { Tabelle } 3 .
$$

Ausbeute an die $P F$ und $C F$, die durch Erhitzen bei $p H 5,0$ und 7,0 extrahiert wurden.

\begin{tabular}{|c|c|c|c|c|c|c|}
\hline \multirow{2}{*}{ Frakt. } & \multirow{2}{*}{$\mathrm{pH}$} & Temp. & \multicolumn{3}{|c|}{ Erhitzung auf $100^{\circ}$} & \multirow{2}{*}{$\begin{array}{l}\text { Verreiben } \\
\text { bei Zim- } \\
\text { mertemp. }\end{array}$} \\
\hline & & Zeitdauer & $15 \min$ & $30 \mathrm{~min}$ & $1 \mathrm{~h}$ & \\
\hline \multirow{6}{*}{$\mathrm{PF}$} & \multirow{3}{*}{5,0} & $\begin{array}{l}\text { Trockengewicht der } \\
\text { Bakterien }\end{array}$ & $5,4054 \mathrm{~g}$ & $6,0714 \mathrm{~g}$ & $6,6543 \mathrm{~g}$ & $\zeta$ \\
\hline & & Ausbeute & $0,06 \mathrm{~g}$ & $0,2295 \mathrm{~g}$ & $0,2695 \mathrm{~g}$ & ノ \\
\hline & & $\begin{array}{l}\text { Prozentsatz der Ausbeute } \\
\text { für Trockengewicht }\end{array}$ & $1,11 \%$ & $3,78 \%$ & $4,05 \%$ &  \\
\hline & \multirow{3}{*}{7,0} & $\begin{array}{l}\text { Trockengewicht der } \\
\text { Bakterien }\end{array}$ & $5,2055 \mathrm{~g}$ & $6,0294 \mathrm{~g}$ & $6,6468 \mathrm{~g}$ & $1,3947 \mathrm{~g}$ \\
\hline & & Ausbeate & $0,3545 \mathrm{~g}$ & $0,5884 \mathrm{~g}$ & $0,4849 \mathrm{~g}$ & $1,1325 \mathrm{~g}$ \\
\hline & & $\begin{array}{l}\text { Prozentsatz der Ausbeute } \\
\text { für Trockengewicht }\end{array}$ & $6,81 \%$ & $9,76 \%$ & $7,28 \%$ & $8,12 \%$ \\
\hline \multirow{6}{*}{$\mathrm{CF}$} & \multirow{3}{*}{5,0} & $\begin{array}{l}\text { Trockengewicht der } \\
\text { Bakterien }\end{array}$ & $5,4054 \mathrm{~g}$ & $6,0714 \mathrm{~g}$ & $6,6543 \mathrm{~g}$ & $\gamma$ \\
\hline & & Ausbente & $0,1492 \mathrm{~g}$ & $0,4104 \mathrm{~g}$ & $0,39 \mathrm{~g}$ & $\zeta$ \\
\hline & & $\begin{array}{l}\text { Prozentsatz der Ausbeute } \\
\text { für Trockengewicht }\end{array}$ & $2,76 \%$ & $6,76 \%$ & $5,86 \%$ &  \\
\hline & \multirow{3}{*}{7,0} & $\begin{array}{l}\text { Trockengewicht der } \\
\text { Bakterien }\end{array}$ & $5,2055 \mathrm{~g}$ & $6,0294 \mathrm{~g}$ & $6,6468 \mathrm{~g}$ & $1,3947 \mathrm{~g}$ \\
\hline & & Ausbeute & $0,2745 \mathrm{~g}$ & $0,328 \mathrm{~g}$ & $0,3845 \mathrm{~g}$ & $0,509 \mathrm{~g}$ \\
\hline & & $\begin{array}{l}\text { Prozentsatz der Ausbeute } \\
\text { für Trockengewicht }\end{array}$ & $5,27 \%$ & $5,44 \%$ & $5,78 \%$ & $3,65 \%$ \\
\hline
\end{tabular}


1) Die Ausbeute der PF war beim Extrahieren in neutraler Reaktion mit oder ohne Erhitzen grösser als die der durch Erhitzen in saurer Reaktion gewonnenen, die PF erreichte ihr Maximum, wenn sie durch 30 Minuten lange Erhitzen bei $\mathrm{pH}$ 7,0 extrahiert wurde, wogegen die der CF in saurer und neutraler Reaktion keinen wesentlichen Unterschied zeigte, abgesehen davon, dass die Ausbeute der durch 15 Minuten langes Erhitzen bei $\mathrm{pH} 5,0$ isolierten $\mathrm{CF}$ und die der aus verriebenen Bakterien in neutraler Reaktion gewonnenen insbesondere gering war.

2) Das geeignetste Isolierungsverfahren, das eine verhältnismässig grosse Ausbeute der stark giftigen Fraktionen ermöglicht, ist für die PF das Bakterienverreiben bei Zimmertemperatur, und für die $\mathrm{CF}$ ein 15-30-minütiges Erhitzen bei $\mathrm{pH} 5,0$.

\section{Uberdiemit Ammoniumsulfat a usfällbaren Fraktionenvon PF und CF.}

Wie oben erwähnt, gewann $\mathrm{K}$ u ro ya neulich aus CF-Lösung der Typhusbazillen eine durch $2 / 3$ Sättigung mit $\left(\mathrm{NH}_{4}\right)_{2} \mathrm{SO}_{4}$ ausfällbare und für Mäuse sehr toxische Fraktion. Diesen Auszug bezeichnete er seinen chemischen Eigenschaften nach mit „Mukoid“ Fraktion (MF).

Um zu prüfen, ob die Toxität der $\mathrm{PF}$ je nach den Sättigungen des Ammoniumsulfats veränderlich sei oder nicht, wurde eine $1 \%$ Lösung von $\mathrm{PF}$ je $2 \mathrm{ccm}$ in eine Reihe von Glasröhrchen verteilt, $\operatorname{mit}\left(\mathrm{NH}_{4}\right)_{2} \mathrm{SO}_{4}$

Tabelle 4.

Die Giftigkhert der mit Ammoniumsulfat aus der PF Gewonnenen Fralctionen für Mäuse.

\begin{tabular}{|c|c|c|c|}
\hline Fr. & $\left(\mathrm{NH}_{4}\right)_{2} \mathrm{SO}_{4}$ & $\begin{array}{c}\text { Injektions } \\
\text { dosis }\end{array}$ & $\begin{array}{l}\text { Mortalität } \\
\text { der Mänse }\end{array}$ \\
\hline I & $\begin{array}{c}\text { durch } \\
\begin{array}{c}\text { Halbsättigung } \\
\text { gefällt }\end{array}\end{array}$ & $\begin{array}{l}2 \mathrm{mg} \\
1 \mathrm{mg} \\
0,5 \mathrm{mg}\end{array}$ & $\begin{array}{l}3 / 3 \\
0 / 3 \\
0 / 3\end{array}$ \\
\hline II & $\begin{array}{l}\text { dureh } \\
\text { Vollsättigung } \\
\text { gefällt }\end{array}$ & $\begin{array}{l}2 \mathrm{mg} \\
1 \mathrm{mg} \\
0,5 \mathrm{mg}\end{array}$ & $\begin{array}{l}3 / 3 \\
1 / 3 \\
0 / 3\end{array}$ \\
\hline III & $\begin{array}{l}\quad \text { durch } \\
\text { Vollsättigung } \\
\text { nicht gefällt }\end{array}$ & $\begin{array}{l}2 \mathrm{mg} \\
1 \mathrm{mg} \\
0,5 \mathrm{mg}\end{array}$ & $\begin{array}{l}1 / 3 \\
0 / 3 \\
0 / 3\end{array}$ \\
\hline
\end{tabular}

Für die Erklärung siche man Tabelle 2. von $1 / 1$ bis $1 / 10$ stufenweise gesättigt, wobei sich ergab, dass bei mehr als Halbsättigung eine ausgesprochene Fällung erfolgte. Nun wurden $100 \mathrm{ccm}$ einer 1\% PF-Lösung bei Halbsättigung mit Ammoniumsulfat gefällt und nach einem 24stündigen Belassen im Eisschrank abgeschleudert, wodurch eine überstehende Fluissigkeit und ein Niederschlag (PF I) gewonnen wurden.

Diese überstehende Flüssigkeit wurde ferner noch mit 
Ammoniumsulfat voll gesättigt, 24 Stunden lang im Eisschrank aufbewahrt, abzentrifugiert und PF II gewonnen.

Die hierdurch erhaltene Flüssigkeit, die noch bei dieser Sättigung eine unfällbare Fraktion in sich schloss, und die PF I und II, nachdem. diese beiden bei $\mathrm{pH}$ 7,0 zur Auflösung gebracht wurden, dialysierte man für sich alleirr in einer Zellophanmembran gegen fliessendes Leitungswasser so lange, bis die Reaktion auf , $\mathrm{SO}_{4}$ " negativ ausfiel. Alle Dialysate wurden dann eingeengt, zu pH 5,0 gebracht und mit $5 \mathrm{Vol}$. abs. Alkohol versetzt. Die erhaltenen Niederschläge wurden nach dem Waschen mit Alkohol und Äther getrocknet und pulverisiert.

Tabelle 4 unterrichtet über die Toxität dieser Fraktionen. Aus dieser Tabelle ist zu entnehmen, dass entweder die durch Halb- und Vollsättigung fällbare Fraktion, oder durch Vollsättigung unfällbare Fraktion zu keiner Vermehrung der Toxität geführt hat, mit anderen Worten, dass $\mathrm{PF}$ von $\mathrm{St}$. aureus mit $\left(\mathrm{NH}_{4}\right)_{2} \mathrm{SO}_{4}$ nicht fraktionierbar waren.

Reindarstellung von PF und CF.

\section{PF.}

Die zu diesem $Z$ wecke herangezogene $P F$ war die aus verriebenen Bakterien gewonnene.

Eine 1 proz. Lösung dieser Fraktion wurde mittels Essigsäure in geeigneter pH-Zone $(3,2)$ gefällt und zentrifugiert. Nach mehrmalig wiederholter Reinigung des Niederschlags durch Säurefällung und Auflösung in dest. Wasser wurde der letzte Niederschlag mit abs. Alkohol und Äther gewaschen und pulverisiert.

2. Reine Polysaccharidfraktion (PSF) aus CF.

Wie oben gesagt, stellte sich die PF unter toxischen Bestandteilen der Staphylokokken quantitativ wie auch qualitativ als ein Hauptbestandteil dar, im Gegensatz dazu stand ihr aber die CF in Toxität nach, und ihre Ausbeute war auch sehr gering. So wurde CF zur Darstellung von nicht toxischer, reiner Polysaccharidfraktion gebraucht. $\mathrm{Zu}$ diesem Versuche verwendete man solche $\mathrm{CF}$, die durch 15 minütiges Erhitzen auf $100^{\circ}$ bei pH 5,0 extrahiert wurde. Eine 1 proz. Lösung dieser Fraktion wurde nach Ansäuerung mit Essigsäure (pH 3,2) 4 Stunden lang erhitzt, die abgetrennte, überstehende Flüssigkeit bei $\mathrm{pH}$ 7,0 in einer Zellophanmembran eine Woche lang dialysiert und dieses Dialysat nochmals bei $\mathrm{pH} 7,0$ bei Unterdruck und niedriger 
Temperatur konzentriert und nach Ansäuerung durch Zusatz von 3 Raumteilen abs. Alkohol gefällt. Dex so erhaltene Niederschlag wurde dann weiter nach dem Waschen mit Alkohol und Äther wie oben, zur Aufbewahrung pulverisiert. Um den alkohollöslichen Anteil zu beseitigen wurde dann das Pulver in 5 Raumteile abs. Alkohol auf $100^{\circ} \mathrm{im}$ Wasserbad umgerührt, abgekühlt und zentrifugiert. Nachdem sich dieses Verfahren 3 mal wiederholt hatte, wurde der Niederschlag mit 5 Vol. Chloroform mitunter geschüttelt, nach 1 tägigem Stehenlassen wieder abgeschleudert und der so gewonnene Niederschlag zu Pulver zerrieben. Er ist die sog. reine „PSF“.

\section{Chemische Eigenschaften von PF und CF.} hin.

Man prüfte rein dargestellte PF und CF auf ihre chemischen Eigenschaften

A. PF.

Diese Fraktion ist leicht graues, weissliches Pulver und ihre Wassersuspension zeigt schwach sauere Reaktion. Das Pulver ist bei $\mathrm{pH} 7,0$ wasserlöslich und nimmt dünn gelblichen Farbenton an.

Tabelle 5 .

$N_{-}, P_{-}$, Asche-, und Zuckergehalt d. $P F$ und $C F$.

\begin{tabular}{c|c|c|c}
\hline Analyse & PF & CF \\
\hline $\mathrm{N}$ & $11,02 \%$ & $3,52 \%$ \\
\hline $\mathbf{P}$ & $0 \mathrm{~h}$ & $3,14 \%$ & $7,1 \%$ \\
\hline & $1 \frac{1}{2} \mathrm{~h}$ & $8,36 \%$ & $25,32 \%$ \\
\hline $\begin{array}{c}\text { Reduzierende } \\
\text { Substanz } \\
\text { nach der } \\
\text { Hydrolyse } \\
\text { wäbrend }\end{array}$ & $3 \frac{1}{2} \mathrm{~h}$ & $8,00 \%$ & $27,12 \%$ \\
\cline { 2 - 4 } & $4 \frac{1}{2} \mathrm{~h}$ & $7,52 \%$ & $28,5 \%$ \\
\cline { 2 - 4 } & $6 \mathrm{~h}$ & $7,44 \%$ & $18,2 \%$ \\
\hline Asche & $2,5 \%$ & $21,5 \%$ \\
\hline
\end{tabular}

Die Eiweiss- und Polysaccharidreaktionen mit einer 1 proz. PF-Lösung ergaben folgende Resultate:-

1) Farbenreaktion:

1. Biuret-R.

2. Mill on sche R.

3. Pauly sche Diazoreaktion

4. Xanthoprotein- $R$

5. Sakagutische-R +

6. Hopkins-Colesche $\mathrm{R}+$

7. Molis chsche $\mathrm{R}+$

8. Glukosamin-R +

9. Phloroglobin-HCl-R +

10. Orcin-HCl-R.

2) Koagulationsversuch.

Die Lösung koaguliert durch Erhitzen in schwach saurer Reaktion, zeigt aber in neutraler Reaktion eine spärliche Trübung. Hellersche Probe +. Sie ist auch durch Alkohol fällbar.

3) Präzipitationsversuch.

Basische Bleiazetat, $\mathrm{FeCl}_{3}$, Phosphorwolframsäure, Tanninsäure, Sulfosalicylsäure und 25 proz. Quecksilberazetat erzeugen einen starken Niedersch- 
lag; PF-Lösung reduzierte in statu Fehling sche Reagenz nicht, es reduziert nach Hydrolyse mit 3 proz. Salzsäure während 3 Stunden nur spurweise.

B. CF.

Die rein dargestellte CF, d. h. PSF bietet weissliches Pulver dar und verhält sich ebenso gleich im Wasser wie die PF, die Lösung ist leicht opaleszent.

1 proz. Lösung ergab folgende Resultate:-

1. Molischsche $R$ \#

3. Orcin-HCl-R \#

2. Phloroglucin-HCl-R

m

5. Biuret-R

4. Glukosamin-R

7. Sulfosalicylsäure erzeugt eine leichte weissliche Trübung, führt aber niemals zur Fällung.

8. Die Fraktion reduziert in statu Feh ling sches Reagenz nicht, reduziert aber nach 3stündiger Hydrolyse mit 3 proz. Salzsäure sehr stark.

C. Analyse der PF und CF.

Zwecks quantitativer Bestimmung von Stickstoff, Phosphor und reduzierender Substanz vor wie nach der Hydrolyse, und Asche wurden folgende Wege eingeschlagen:

N durch Halbmikrokjeldahl, P durch Lohmann-Jendrassik und Reduktionskraft durch Haged orn-Jensen (Tabelle 5).

\section{Schlussfolgerungen.}

1) Aus einem Fall von Ohrkarbunkel wurde ein Stamm von Staphylococcus aureus auf gewöhnlichen Agarnährböden gezüchtet, angesammelt und in destilliertem Wasser aufgeschwemmt. Einerseits durch 15,30 und 60 minütiges Erhitzen im Wasserbad auf $100^{\circ}$ bei $\mathrm{pH} 7,0$ oder 5,0 und andererseits durch Wasserextrahieren aus verriebenen Bakterien bei $\mathrm{pH} 7,0$ konnten spezifische Substanzen isoliert werden.

2) Wurde diesen Extrakten Essigsäure zugesetzt, so trat in ihrem isoelektritischen Punkt ein Eiweissniederschlag auf. Der so erhaltene Niederschlag wurde mit verdünntem Alkali und Wasser gelöst und nochmals mit Essigsäure gefällt. Durch das Wiederholen dieser Manipulation wurde das Säurepräzipitat, d. h. die PF-Fraktion gewonnen.

3) Die von der PF abgesonderte überstehende Flüssigkeit wurde angesammelt, bei niedriger Temperatur eingeengt und nach Beseitigung von übrig bleibender PF mittels Essigsäure dialysiert. Das Dialysat wurde erneut konzentriert und bei $\mathrm{pH}$ 5,0 mit 3 Volumen abs. Alkohol gefällt. Nach mehrmaligem Wiederholen der Reinigung wurde die säurelösliche Fraktion (Kohlenhydrat) CF gewonnen.

4) Die mit PF-Lösung angestellte Eiweissreaktion erwies sich fast ganz als positiv. Ihr Stickstoff betrug 11,02\%, Phosphor 1,53\%, reduzierende Substanz $8,36 \%$ und Asche 2,5\%. Demnach gehört die PF einwandrei dem Eiweiss an. 
5) Bei CF-Lösung fielen Phloroglucin-, Orcin- und Molis c hsche Probe positiv aus, Biuret- und Hopkins-Colesche Probe hingegen negative. Sie enthielt 3,52\% Stickstoff, 1,64\% Phosphor, 28,5\% reduzierende Substanz als höchsten Wert nach der Hydrolyse und 21,5\% Asche. Also gehört CF zu einer Art Polysaccharid.

6) Bei der aus unerhitzt verriebenen Bakterien gewonnenen PF stellen $2 \mathrm{mg}$ eine für Mäuse von $10 \mathrm{~g}$ Gewicht sicher tödliche Dosis dar; darauf folgten an Toxität die durch 15 minütiges Erhitzen auf $100^{\circ}$ bei pH 5,0 und 7,0 gewonnene(M.L.D. $3 \mathrm{mg}$ ). Durch 15 minütiges Erhitzen auf $100^{\circ}$ bei $\mathrm{pH} 5,0$ oder 7,0 wurde auch die giftigste CF ausgezogen, jedoch betrug ihre M.L.D. für Mäuse nicht mehr als $3 \mathrm{mg}$.

7) Die Hitzebehandlung bei $\mathrm{pH} 7,0$ erbrachte im allgemeinen eine grössere Menge PF als die bei $\mathrm{pH}$ 5,0. Die Menge der bei $\mathrm{pH} \mathrm{7,0}$ isolierten PF betrug im Mittelwert auf gesamte Tockenbakterien bezogen 7,99\%, bei pH 5,0 dagegen 2,98\%. Für die Gewinnung der CF war die Erhitzung bei $\mathrm{pH} 5,0$ am geeignetsten vom Standpunkt der Ausbeute und Toxität. Daraus liess sich schliessen, dass das Verreiben der Bakterienleiber bei $\mathrm{pH}$ 7,0 für die Gewinnung der PF und das Erhitzen in schwach saurer Reaktion für die der CF sehr empfehlenswert waren.

8) Es gelang nicht, mittels Ammonsulfat die toxischen PF und $\mathrm{CF}$ in noch giftigere Fraktionen zu zerteilen.

\section{Schrifttum.}

(1) Burnet, F. M., J. Path. and Bact., 1929, 32, 717.

(2) Burnet, F. M., Ibid., 1930, 33, 1.

(3) Burnet, F. M., Ibid., 1932, 35, 477.

(4) Avery, M., und Heidelberger, M. D., J. Exp. Med., 1923, 38, 73.

(5) Julianelle, L. A. und Wieghard, C. W., Proc. Soc. Exp. Biol. a. Med., 1934, 31, 947.

(6) Julianelle, L. A., und Wiegh ard, C. W., J. Exp. Med., 1935, 62, 31.

(7) Y at o, M., J. Oriental Med., 1935, 22, 157.

(8) Robert, P., Arch. f. Dermat., 1937, 175, 530.

(9) Kod a ma, M. und Nisi y a ma, S., Kitasato Arch. exp. Med., 1938, 15, 241.

(10) Verwey, W. F., J. Exp. Med., 1940, 71, 635.

(11) Ku roya, M., Oshi o, K. und Hu, P. K., Tohoku J. Exp. Med., 1940, 38, 167.

(12) Kuroya, M. und Koizumi, K., Tohoku J. Exp. Med., 1940, 38, 305.

(13) Ku roga, M. und Koi z u mi, K., Tohoku Igaku Zassi, 1941, 29, 231.

(14) K o i z u mi, K., Ibid., 1942, 30, 243.

(15) Y a m a moto, K., Tohoku J. Exp. Med, 1941, 4l, 70.

(16) Y a m a m o t o, K., Ibid., $1942,42,198$

(17) Suga numa, T., Ibid., 1940, 38, 554.

(18) Suganuma, T., Ibid., 1940, 39, 180.

(19) Suganuma, T., Ibid., 1941, 40, 475 .

(20) Suganuma, T., Ibid., 1941, 40, 492. 